casual reader of his letter would form the impression that, but for Edinburgh, ipecacuanha would not have been introduced into India, and that, consequently, the Kew establishment cannot be relied upon for the dissemination of useful plants among the British possessions abroad, which is, I imagine, one of "the works and applications for which a nation provides and supports its collections of living plants."

My report having been quoted with such an object, I wish to state that the success of ipecacuanha cultivation in India had been practically settled before any of the plants propagated at Edinburgh had arrived in that country. In the year 1866, and long before Government had begun to show any official interest in the matter, Dr. Hooker sent an ipecacuanha plant to the Calcutta Botanical Garden, the offspring of which, in Sikkim, amounted, in the month of September, $187 \mathrm{I}$, to nearly $400-$ quite a sufficient number to acsure a successful start to propagation on a large scale. The subsequent arrival in India of considerable supplies from Edinburgh has, indeed, made assurance doubly sure; but the fact remains that to Dr. Hooker is India indebted for the first beginning of this important cultivation.

If the establishment at Kew stood in any need of a testimonial as to the valuable assistance rendered by it in the introduction into India of plants of economic or horticultural interest, it would not be difficult to furnish a list sufficient to fill a good many columns of NATURE of the names of plants and seeds sent-many of them quite unsolicited-from Kew to Calcutta within the last ten years, to go no farther back.

\section{George KInG,}

Superintendent Calcutta Botanical Garden

Nice, Nov. 26

\section{The Great Meteoric Shower}

As you will most probably have received from many other correspondents a general description of the magnificent spectacle on last Wednesday evening, I will confine my remarks principaily to those observations which bear directly on the most important point at issue, viz., whether this meteor stream can be identified with the well-known comet of Biela. Having searched, during the autumn, on every available occasion for a glimpse at the approaching comet, and the almost unvarying cloudiness of the early morning sky having rendered even the negative value of the observations well-nigh useless, I read with delight the prediction of Dr. Weiss, and felt the greatest interest in its fulfilment.

Immediately I had noticed that 2 meteoric shower was in pro. gress on the evening of the 27 th, I directed the two assistants of the observatory, who have had considerable experience in tracing the paths of meteors during the last few years, to devote their whole attention to the accurate determination of the radiant point. In the meantime, with the assistance of three of the students of the philosophy class and of the meteorological assistants of the observatory, I noted the rate per minute, the velocity, direction, magnitude, \&c., of the falling bodies.

The Radiant was found to be on the line joining $\gamma$ and $51 \mathrm{An}$ dromedæ, and twice as far from $5 \mathrm{x}$ as from $\gamma$. This gives as the R.A., $26^{\circ} 37^{\prime}$, and N. Decl. $43^{\circ} 48^{\prime}$, agreeing very well with the prediction.

The Epoch is somewhat in advance of that predicted; but this cannot be wondered at, as the comet has not been seen since I852, and, in three complete revolutions round its orbit, it could scarcely have been expected not to have been subjected to considerable unknown perturbation, either from planets whose masses are imperfectly known, or perhaps from some neighbouring meteor-stream.

The time of the maximum was about 8 h. Iom. P.M., but the numbers did not much diminish before 9 P.M., G.M.T. Between $8 \mathrm{~b} .47 \mathrm{~m}$. $30 \mathrm{~s}$. and $9 \mathrm{~h}$. om, the computer of the observatory counted $5 \mathrm{I} 2$, which gives 40 per minute for one observer, and therefore at least 100 per minute invisible. From 9 to 10 o'clock, at which time the sky became clouded, and remained so till morn. ing, the mean rate was about 53 per minute, and almost constant from minute to minute, though varying much during each minute. At certain moments they were exceedingly numerous.
Thus, at $9 \mathrm{~h} .19 \mathrm{~m}$. nine appeared at the same instant at a point near $\beta$ Andromedæ.

A very peculiar feature of the display was the parallel motion of many stars that became visible at the same time. Thus, at $9 \mathrm{~h} .16 \mathrm{~m}$. five burst out close by $\gamma$ Andromedæe, and travelled eastward together; at 9.25 four went together from $\gamma$ Andromedx to the Pleiades.

More than nine-tenths of the meteors were very faint, and the larger ones seldom attained to any very considerable magnitude. Most had tails; the almost invariable colour being a white star with a greenish-blue trail. The tails of those falling S.E. were observed to bend somewhat towarus the $E$, and to be straight only during the first half of their path. The ratio of the numbers falling S.E., to those falling N.W., was as 3 to 2 , but this excess may in part be accounted for by the position of the Radiant. More of the larger meteors went S. than N., and more W. than E. The track of the larger bodies rarely, if ever, exceeded $50^{\circ}$, and their velocity was very noticeably less than that of the I3th and I4th November shower, as might be expected, if their absolute velocities are comparable, the Radiant tor November 27 being so far removed from the apex of the earth's way.

Stonyhurst Observatory, Dec. I

S. J. PERRY

A LTHOUGH it is probable that you will reeeive full accounts of the meteoric shower of Wednesday, November 27 , yet the following notes, imperfect though they be, may have some interest. $Y$ was prevented by indisposition from observing it myself, but the numbers were noted by Captain Brinkley, grandson of the great astronomer, and his sons :--

"Mr. Charles B., at 3 P M., observed a bright meteor; Capt. B., at 4.35 , another; at 5.20 the young men came in to announce an extraordinary display; and Capt. B. noted 34 in Im. 30s.; Capt. B., looking north at 5.40 , marked 95 in $5 \mathrm{~m}$. ; Mr. John B., looking south at same time, marked 147 in $5 \mathrm{~m}$. ; the radiant point was a little S.E. of the zenith; Mr. J. B., at 9 , marked 26 in Im. ; Capt. B., at 12 , marked only 7 in $5 \mathrm{~m}$. Many were large, and left trains."

It was remarked that the night was unusually light, while clear. A very thick fog appeared before the dawn of Thursday.

Castleknock, Dublin, Nov, 29

T. R, R.

A FIN display of shooting stars was observed here on Wed. nesday, 27 th inst. I first noticed them at 7.20 P.M., Greenwich time, and watched them till about 8 , when the sky became obscured. They were occasionally seen again till 9.30. When first observed they appeared to radiate from the zenith, and to be more numerous towards the north-west and south-west; many passed over the constellation Cygnus.

St. David's College, Lampeter, Nov, 30

DURING the recent star-shower, my attention was given espe. cially to observations connected with the fight of individual meteors. As on many previous occasions in the presence of rare natural phenomena, I was keenly mortified with the deficiency of my own scientific training; but $I$ send a few gleanings, if perchance a useful grain can be found amongst them. The brightness obviously increased with the distance traversed, but in many cases no increase of brightness was perceptible for the first third of the course. The extinction was not instantaneous but only very rapid, the distance traversed towards extinction being perceptible though very small; perhaps because the velocity seemed to diminish as the brightness increased. The train in many instances was forked, being brightest on its edges, the luminosity of which lasted for some time after the intermediate space was dark. This seems incompatible with the hypothesis that the train is a mere optical result, or tliat the brightness of the train arises, as in lightning, from incandescent particles of the atmosphere. In one or two instances the bright. ness of the train was granular, resembling the light of a partially resolved nebula, or of the Galaxy. In a few instances the paths of the meteors appeared to show remarkable deflections. One, notably, at $6 \mathrm{~h} .25 \mathrm{~m}$, close to Vega, resembled an "S" drawn out nearly straight.

The course of a body passing with great velocity though an elastic medium tends to follow the direction of least resistance. It is only in poetry that

$$
\text { "The lightning falls with never a jag." }
$$

